Musées, Patrimoine et Culture scientifiques et techniques

$156 \mid 2014$

novembre-décembre 2014

\title{
Infestations en exposition permanente : un exemple à la Grande Galerie de l'Évolution
}

Benoît Gayral et Jacques Cuisin

\section{OpenEdition \\ Journals}

Édition électronique

URL : http://journals.openedition.org/ocim/1464

DOI : $10.4000 /$ ocim. 1464

ISSN : 2108-646X

Éditeur

OCIM

Édition imprimée

Date de publication : 1 novembre 2014

Pagination : $24-31$

ISSN : 0994-1908

Référence électronique

Benoît Gayral et Jacques Cuisin, «Infestations en exposition permanente : un exemple à la Grande Galerie de l'Évolution 》, La Lettre de l'OCIM [En ligne], 156 | 2014, mis en ligne le 01 novembre 2016, consulté le 19 avril 2019. URL : http://journals.openedition.org/ocim/1464 ; DOI : 10.4000/ocim.1464

Ce document a été généré automatiquement le 19 avril 2019

Tous droits réservés 


\section{Infestations en exposition permanente : un exemple à la Grande Galerie de l'Évolution}

Benoît Gayral et Jacques Cuisin

La caravane africaine de la Grande Galerie de l'Évolution après rénovation.

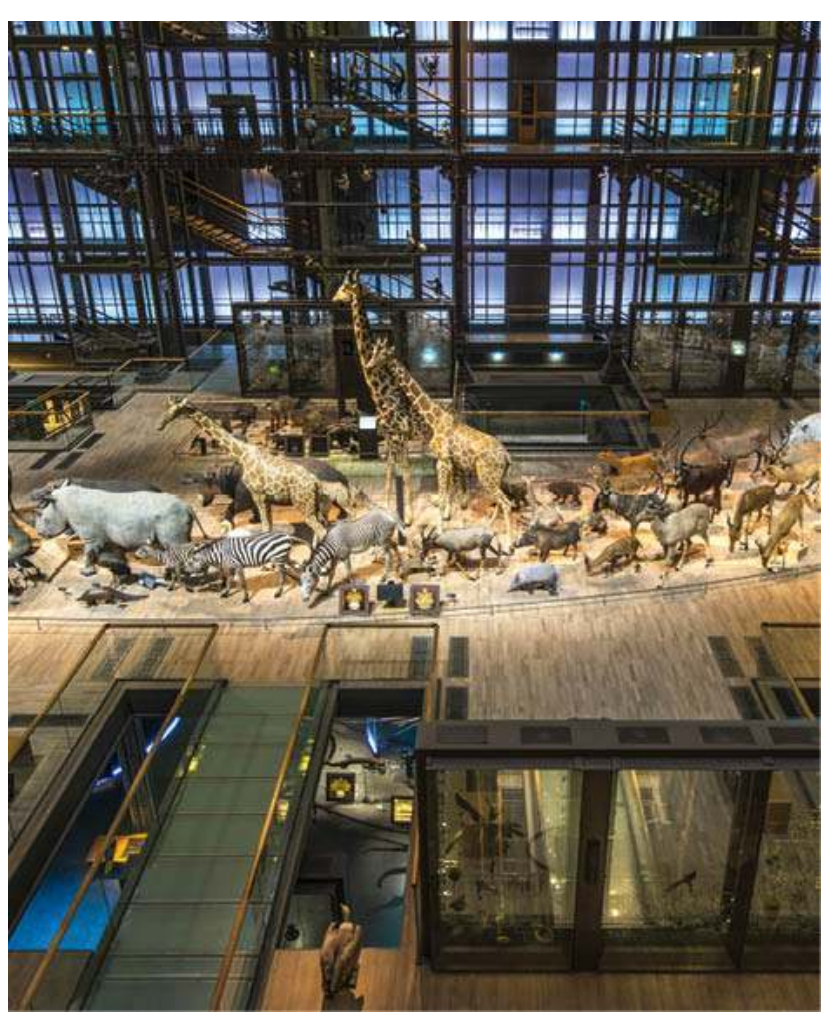

(C) MNHN/B. Jay 
1 Ce travail résulte d'un stage effectué entre mars et juin 2013 dans le cadre du Master "Muséologie : Sciences, Cultures et Sociétés" du Muséum national d'Histoire naturelle.

2 La problématique de ce stage était de répondre à certaines hypothèses portant sur les origines des infestations d'insectes kératophages (Tineidae essentiellement) à l'intérieur de la Grande Galerie de l'Évolution (GGE). Cette galerie a connu, presque dès son ouverture en 1994, des invasions de mites, invasions alors assez espacées dans le temps et d'ampleur aléatoire, mais plutôt faibles. Les traitements chimiques appliqués dès cette époque n'ont toutefois jamais permis d'éradiquer totalement l'espèce (Maigret et al. 2002).

Or, depuis quelques années, le nombre et les dommages occasionnés semblent s'accroître malgré des protocoles de suivi et de lutte mis en place depuis près de 20 ans. Les démarches de suivi, régulièrement reprises et techniquement améliorées, n'ont jamais complètement pu déboucher sur une politique de traitement satisfaisante, puisqu'il faut constamment l'adapter. Ce problème d'infestation, devenu récurrent, constitue une source d'inquiétude pour les chargés de conservation car, chaque année, des spécimens sont endommagés et certains retirés de l'exposition permanente au moins temporairement, pour restauration.

4 Il importait donc de dresser un état des lieux le plus objectif possible, afin d'identifier les causes de ces infestations répétées et, ainsi de préparer le travail de mise en place d'un protocole de surveillance et de lutte (Fohrer, 2011). Il a donc fallu poser et répondre à de nombreuses questions sur les facteurs pouvant être à l'origine de la prolifération de ces insectes, mais aussi tester des hypothèses, confirmer ou infirmer certaines analyses des phénomènes d'infestation. Si la question des ravageurs kératophages est constante depuis 1994 (Maigret et al., 2002), l'anniversaire des 20 ans de la Grande Galerie en 2014 motivait un surcroît d'efforts. Le présent article traite essentiellement des Tineidae, mais d'autres familles d'insectes kératophages sont évoquées.

\section{Protocoles}

5 Afin de cerner et de comprendre la nature et l'ampleur des phénomènes d'infestation, plusieurs angles de recherche ont été définis : exploiter les archives, bénéficier de la mémoire des acteurs de la GGE à son ouverture encore en activité, des données collectées actuellement, enquêter sur le terrain par le biais de piégeage ou de collecte directe. Cette stratégie, destinée à recueillir un maximum de données chiffrées et localisées, a elle aussi été évolutive au cours du stage. Tous les pièges utilisés au cours de cette étude sont de type "piège à glu", achetés dans le commerce auprès de sociétés spécialisées françaises, le contenu de la capsule de phéromones n'est pas détaillé par le revendeur, qui l'indique comme spécifiquement ciblé pour les Tineolidae. Le principe actif de ces pièges, inclus à la glu, est un pyréthrinoïde de synthèse, type transfluthrine (à $0,20 \%$ ), qui a pu varier selon les marques commerciales (les fournitures ont légèrement changé en 20 ans, toutes n'ont pas été archivées ni achetées avec une fiche technique depuis 1994). Ce type de piège a cependant beaucoup évolué depuis 20 ans, puisqu'il ne contient plus aujourd'hui de produit actif de synthèse, mais des produits "verts".

6 À la Grande Galerie, ces pièges sont toujours disposés hors vitrine, en des endroits choisis pour établir un maillage ou en des endroits très stratégiques (entrées des flux de marchandises, ou autour de l'espace café, par exemple, ou autour de spécimens disposés 
hors vitrines, dans la caravane africaine...). La notion d'un ratio entre nombre de pièges et volume à surveiller n'est pas facilement applicable dans un bâtiment de plus de $97000 \mathrm{~m} 3$ accessible au public... Le maillage, même lâche, a pour but de surveiller des secteurs qui semblent plus sensibles que d'autres en fonction des risques identifiés. Ces pièges à phéromones sont systématiquement remplacés tous les 6 mois. Une analyse de leur contenu est effectuée toutes les semaines. Un changement récent de protocole (septembre 2014) a cependant vu ce type de piégeage remis en cause, non à cause de sa performance, mais plus à cause de la disparition annoncée des postes occupés par les agents en charge des relevés.

\section{Enquête historique : 1996-2008}

7 Les données concernant les mites de la GGE semblent sporadiques pour la période considérée, même si une partie des archives de la maintenance des collections de cet espace a malencontreusement disparu, probablement à la suite des trop nombreux déménagements (P.-Y. Gagnier, communication personnelle). Cela étant, une reconstitution fondée sur des données éparses laisse apparaître une situation d'infestations qui représentaient déjà un souci dès les premières années d'exploitation de la GGE : sur un flamant rose (juillet 1995); sur deux cerfs muntjacs, près de la vitrine iules et dans la caravane africaine vers l'ascenseur desservant les locaux administratifs (mars 1996) ; à proximité des locaux du service de l'action culturelle (venant de la vitrine des microcèbes) et dans les vestiaires publics (le 24 avril 1996, date de la pose de pièges à phéromones).

Les vitrines anciennes au niveau III de la Grande Galerie de l'Évolution.

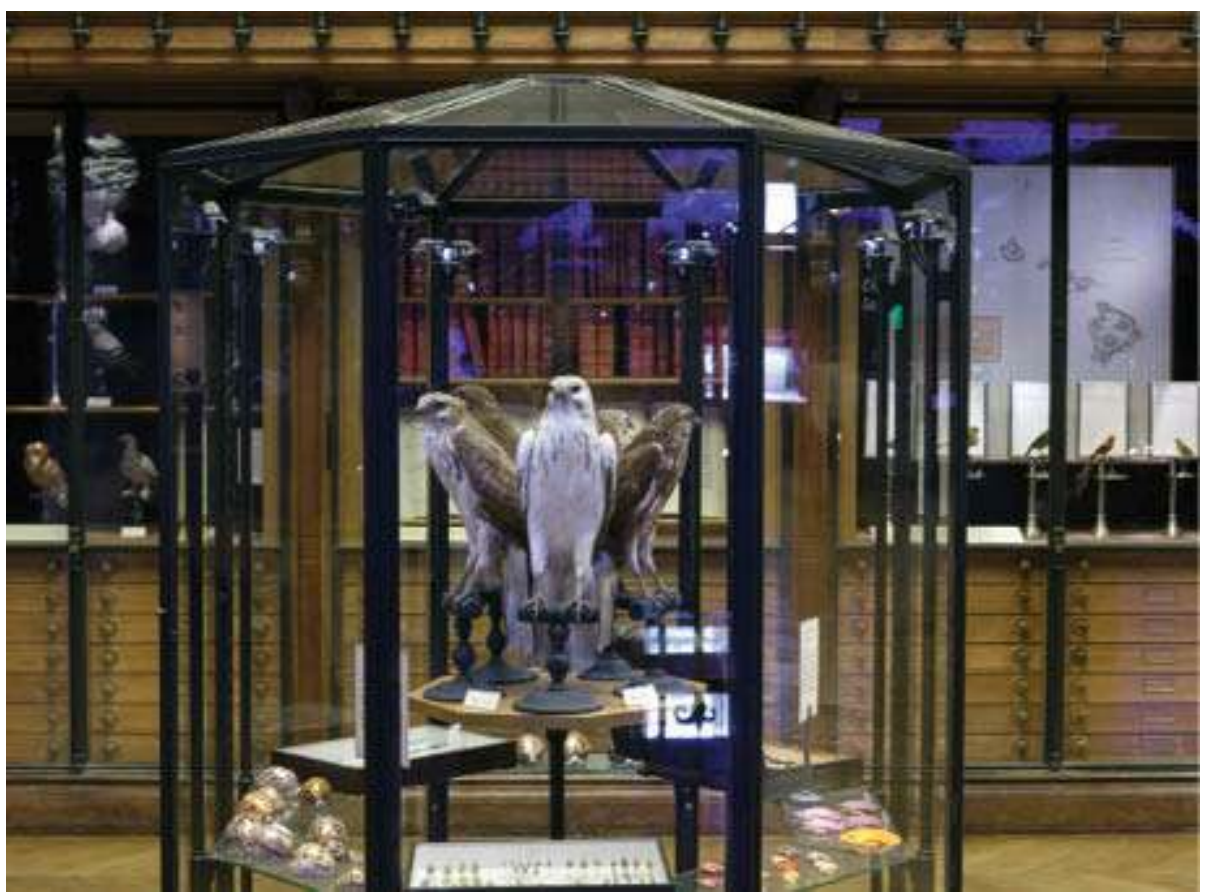

(c) MNHN/B. Faye

La surveillance s'est par la suite affinée et a permis l'établissement de données chiffrées. Entre avril 1996 et mars 1997, 98 Tineola bisselliella ont été capturées à tous les niveaux 
de la GGE, de R0 à R+3 (Maigret et al., 2002). La campagne de novembre 1997-juin 1998 piégera 114 mites en 29 semaines. Toutefois, l'identification des insectes a prouvé la présence de la mite des poulaillers (Monopis crocicapitella), due aux pigeons qui nichaient sous la toiture.

9 À la fin de l'année 1999, après plusieurs désinsectisations successives, les captures ont très nettement diminué. Le protocole prévoyait à cette époque un traitement par pulvérisation bisanuelle avec un produit contenant pyréthrine, diméthrine et perméthrine. Tous les 2 ans, une pulvérisation au Dichlorvos ${ }^{\oplus}$, alors autorisé, complétait cette première salve. De plus, toutes les surfaces non accessibles aux visiteurs (gaines technique et sous plancher) étaient traitées tous les 2 ans avec du Cislin ${ }^{\circledast}$ (deltaméthrine ; ce produit est aujourd'hui dénommé K-Othrine SC25, www.pestcontrol-expert.com). Les vitrines étaient protégées de l'intérieur par des plaquettes au début insecticides, par la suite répulsives (citronnelle), avec changement annuel (F. Jullien, communication personnelle). Le coût humain (effectifs) ou financier (budget) très conséquent que cela impliquait ne peut plus être aujourd'hui assumé.

10 Entre 1999 et 2006, aucun relevé de mites n'est connu, mais cette période correspond au manque d'archives évoqué précédemment. À partir de 2006, les données sont collectées au moyen de 14 pièges à phéromones (voir tableau ci-dessous) qui indiquent la prise non seulement de mites, mais aussi de quelques coléoptères (Anobiidae) et diptères.

11 Les relevés des 6 premiers mois de 2008 n'indiquent aucune capture de mite, ce qui est surprenant au vu de l'historique et de l'actualité des infestations. Il faut attendre l'intervention de la Direction des Collections, qui prend en charge les collections exposées à partir de 2009, pour qu'une surveillance reprenne progressivement, mais là encore, des manques ponctuels se remarquent (le classeur de surveillance des infestations 2011-2012 a lui aussi disparu). Le type de traitement annuel est le même que précédemment: produit actif à base de pyréthrines de synthèse, dispersé au moyen de bombes à diffusion aérosol, le traitement s'effectuant sur plusieurs jours. Les pyréthrines ne sont plus les mêmes qu'à l'époque de l'ouverture de la GGE. Sa technicité sera changée en 2010 (fumigation sèche appliquée en une seule journée, la molécule du principe actif reste la même). En 2013 et 2014, le traitement a consisté en dispersion AquaPy ${ }^{\circledast}$ par micronébulisation (Préviato, communication personnelle); le principe actif du produit est indiqué comme étant composé de pyréthrines naturelles extraites de fleurs de Pyrethrum cinerariae-folium (pestcontrol-expert.com). 


\begin{tabular}{|c|c|c|c|c|c|c|c|c|c|}
\hline $\begin{array}{c}\text { Piège } \\
n^{\circ}\end{array}$ & Localisation & $\begin{array}{l}\text { sept. } \\
2006\end{array}$ & $\begin{array}{c}\text { oct. } \\
2006\end{array}$ & $\begin{array}{l}\text { nov. } \\
2006\end{array}$ & $\begin{array}{l}\text { déc. } \\
2007\end{array}$ & $\begin{array}{l}\text { janv. } \\
2007\end{array}$ & $\begin{array}{c}\text { fév. } \\
2007\end{array}$ & $\begin{array}{l}\text { mars } \\
2007\end{array}$ & $\begin{array}{c}\text { avril } \\
2007\end{array}$ \\
\hline M. 1 & Niveau marin & & \multirow{14}{*}{ 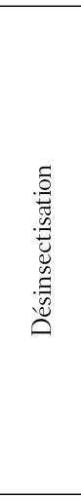 } & 5 & 7 & 7 & 7 & 7 & 7 \\
\hline M. 2 & Animalerie & & & & & & & 1 & 1 \\
\hline M. 3 & Escalier raie manta & 1 & & 2 & 3 & 3 & 3 & 3 & 3 \\
\hline M. 4 & Rhino Louis XV & & & & & & & & \\
\hline M. 5 & Muntjacs & & & 1 & 1 & 1 & 1 & 1 & 1 \\
\hline M. 6 & Escalier bac vertébrés & & & 5 & 5 & 5 & 5 & 5 & 5 \\
\hline M. 7 & Gaine RIA & & & 2 & 2 & 2 & 3 & 3 & 3 \\
\hline M. 8 & Domestication & & & & & & & & \\
\hline M. 9 & Big-Bang & & & & & & 1 & 1 & 1 \\
\hline M. 10 & Ascenseurs & & & & & & & & 1 \\
\hline M. 11 & Vigogne & & & & & & & 1 & 1 \\
\hline M. 12 & Médiathèque & 1 & & 4 & 5 & 5 & 5 & disparu & disparu \\
\hline M. 13 & Action pédagogique & & & & & & 1 & disparu & disparu \\
\hline M. 14 & Cafétéria & & & & & & & & \\
\hline & Total & 2 & & 19 & 23 & 23 & 26 & 22 & 23 \\
\hline
\end{tabular}

La méthodologie mise en œuvre dès 2006 comporte 14 points de piégeage, dont la synthèse est faite chaque mois. La désinsectisation de 2006 semble avoir fait bouger les populations d'insectes sans les éradiquer ( 17 captures en novembre pour seulement 2 en octobre). Intriguant sur les relevés de 2006 ce constat est systématiquement fait depuis 2010 : le nombre de captures est toujours plus important immédiatement après traitement qu'avant, ouvrant la voie à une population d'insectes en cours de pérennisation.

\section{L'année 2013}

12 L'ampleur des locaux de la GGE, ainsi que la complexité climatique du lieu font que la résolution des infestations n'est vraiment pas une tâche facile. Des pièges supplémentaires ont été placés dans des endroits non encore testés, en fonction de l'identification du risque d'infestation du lieu. L'ensemble du dispositif se monte ainsi à 20 pièges. Outre une répartition spatiale cohérente, leur emplacement a été choisi en fonction des flux d'air, de visiteurs, des ouvertures, du recouvrement des zones d'attractivité entre elles.

Entre le 21 mars 2013, date de pose des nouveaux pièges, et le 31 mai de la même année, date des derniers relevés, plus de 500 mites "nouvelles" ont été capturées. L'explosion populationnelle tient à l'ajout de nouveaux pièges qui augmente mécaniquement le nombre de captures dans des zones jusqu'alors moins surveillées, cependant, une seconde hausse spectaculaire se situe entre le 2 et le 10 mai, 71 mites supplémentaires sont capturées, soit $87 \%$ d'augmentation par rapport à la semaine précédente. Au total, le nombre de captures, de 34 en début d'étude (21 mars 2013, pièges à phéromones et glu) atteint 557 en fin d'étude (31 mai 2013).

14 À l'évidence, l'ensemble de la Grande Galerie de l'Évolution est désormais infesté de manière permanente. Les capacités de déplacement de ces insectes étant réduites, la galerie peut être considérée comme abritant une population de mites autonome: les larves et les œufs cachés sous les matériaux et débris divers sont protégés de l'action des traitements insecticides (nébulisation) et peuvent se développer sans risques. La prolixité des populations, révélée par le nombre de captures, ne peut de fait s'expliquer que par des conditions de développement favorables. Au nombre de celles-ci, on peut citer: domaine vital, nourriture, conditions climatiques favorables, pression de destruction supportable, et (éventuelle) compétition avec d'autres espèces/groupes populationnels elle aussi supportable. Ces éléments sont à examiner au travers de diverses hypothèses. 


\section{Hypothèse 1 : Le public est-il en cause?}

Le public de la Grande Galerie de l'Évolution, toutes catégories confondues, représente en moyenne sur 20 ans, 500000 visiteurs annuels (643 190 visiteurs en 2006, 545200 en 2009, 738053 en 2011, source : Ministère de la Culture, 2007, 2010, 2013).

L'arctique à la Grande Galerie de l'Évolution.

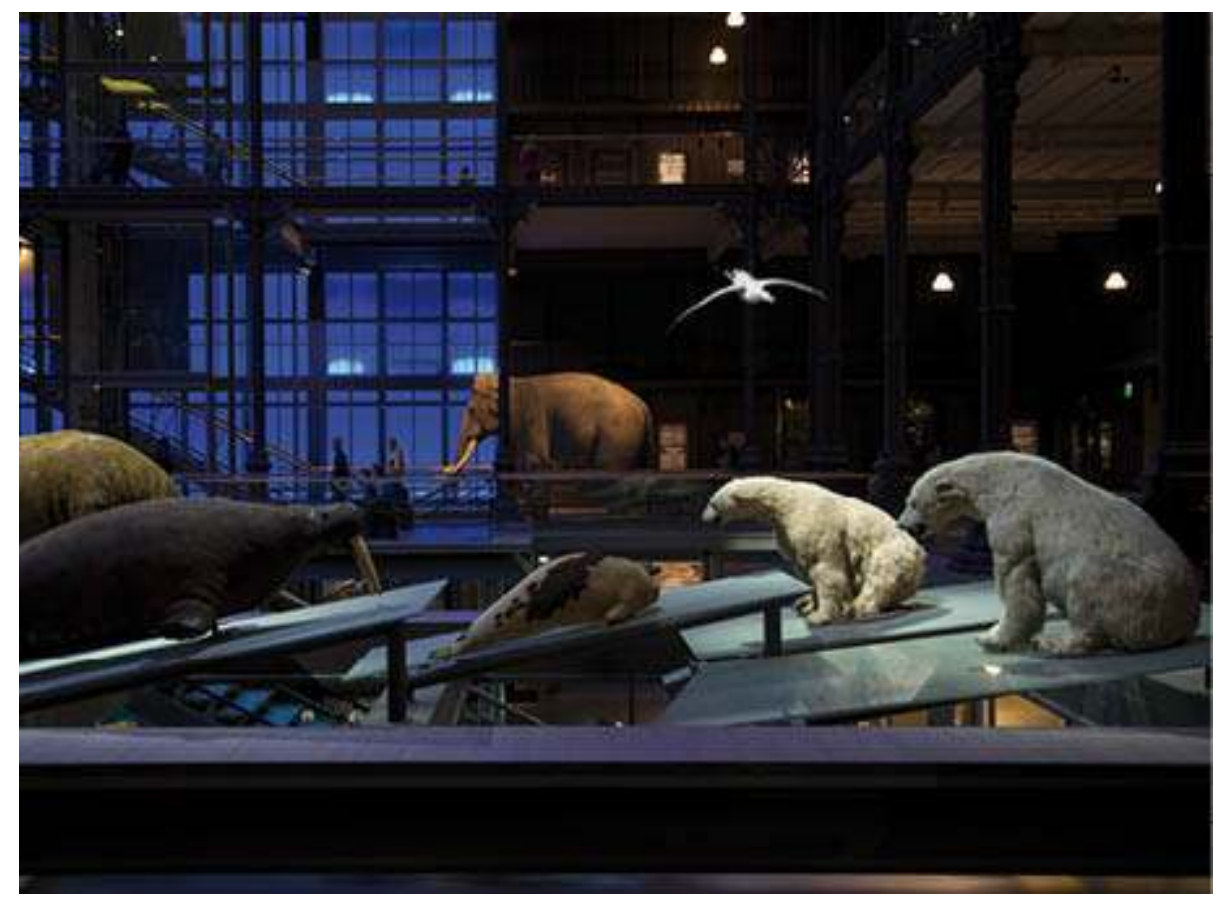

(C) MNHN/B. Faye

Cette hypothèse, émise de manière intuitive il y a quelques années, considère que la fluctuation des populations des mites était liée, au moins pour partie, à la fréquentation du public. Celle-ci est variable en fonction des périodes de l'année, notamment au moment des congés scolaires. Ainsi, si une corrélation éventuelle existe entre fréquentation de la galerie et infestation par les mites, cette dernière devrait être principalement observée pendant ces périodes de l'année, et plus encore pendant les congés de printemps (émergence naturelle post-hivernale et de reproduction) et d'automne.

Dans cette hypothèse, les mites entreraient plutôt sous forme de larves ou d'œufs, à l'instar de ce qui a été observé lors des infestations provoquées par des objets non ou incomplètement décontaminés entrant en exposition temporaire (cas de l'Exposition Himalaya-Tibet, le choc des continents, décembre 2002-novembre 2003). Dans le cas du public et des infestations susceptibles d'entrer par le biais de vêtements (fourrure, manteaux de laine épais, fourrés de duvet...), les preuves sont très difficiles à établir, il ne faut donc garder cette hypothèse que comme un risque possible. Cette hypothèse paraît néanmoins étayée par l'attaque d'un spécimen placé à l'entrée de l'exposition temporaire Nature Vive, dont la prolongation s'est déroulée pendant une période très froide à Paris (janvier 2002), amenant les visiteurs avec ce type de vêtements. 
18 L'hypothèse du public a également été testée en analysant les données de l'observatoire des publics (chiffres de fréquentation). Ainsi, le taux de fréquentation par le grand public est en accord, au 1er semestre 2013, avec les variations saisonnières relevées au cours des derniers exercices d'exploitation. Il n'y a donc pas de pic particulier, ou de phénomène d'infestation que l'on puisse corréler immédiatement à la fréquentation et sans doute en est-il ainsi depuis plusieurs années. Le public peut avoir une influence mais celle-ci est certainement très faible et surtout à peu près indécelable, le public agit comme une "cause silencieuse" des infestations.

19 Cela étant, si l'on considère le public comme un "utilisateur des lieux de manière temporaire", cette catégorie est aussi susceptible de contenir les artistes plaçant des œuvres dans la GGE, comme lors de la 38e édition de la FIAC (octobre-novembre 2011), au cours de laquelle une œuvre réalisée en poussière récoltée dans le métro parisien et insuffisamment traitée a importé une nouvelle population de mites, qui s'est rapidement et massivement prise dans les pièges à phéromones. L'œuvre a été retirée 24 heures après la découverte de l'infestation pour traitement en camion frigorifique, puis contrôlée et replacée.

\section{Hypothèse 2 : Le climat, facteur favorable ou défavorable?}

Une seconde hypothèse de travail est celle de l'influence du climat sur le développement des populations de mites. (Chauvin, 1977). Il s'agit dans ce cas essentiellement du climat intérieur de la GGE. La maîtrise des conditions climatiques de la GGE a pris beaucoup de temps, elle ne s'est pas faite sans problèmes ni à-coups, et nécessite encore aujourd'hui une grande vigilance. Les deux principaux paramètres contrôlés dans la GGE sont le taux d'humidité relative (HR ou hygrométrie) et la température. L'éclairage intérieur de la GGE est artificiel et faible, dans la conception même du parcours muséographique; la luminosité réduite est un facteur favorable à la présence des Tineidae, qui ont une tendance lucifuge.

21 Le climat intérieur de la GGE n'a pas connu de variation accidentelle au cours de la période d'étude et pendant les périodes immédiatement précédentes (les deux derniers accidents climatiques ont eu lieu en 2005 et 2010). Aujourd'hui, les conditions climatiques sont stables, et comprises dans les recommandations, soit $55 \%$ d'HR avec un seuil de tolérance de $\pm 5 \%$ sur 24 heures, $22^{\circ} \mathrm{C}$ en hiver et $24^{\circ} \mathrm{C}$ en été avec un seuil de tolérance de $\pm 3^{\circ} \mathrm{C}$, toujours sur 24 heures. Ces conditions climatiques, globalement stables, offrent toute l'année une température supérieure aux minimales de développement de Tineola bisselliella (Chauvin et Vannier, 1997; Strang, 1992), mais ces standards s'accordent d'abord avec le confort de visite du public.

Les courbes d'hygrométrie allant de début janvier à fin mai 2013 montrent que le taux d'hygrométrie le plus stable se trouve au niveau 2, les plus fortes fluctuations atteignant régulièrement des taux de $45 \%$, voire moins, se trouvant au niveau 0 . Cette zone de la galerie est en fait soumise à l'influence des conditions extérieures en raison de l'ouverture et de la fermeture très fréquente des portes. Ces variations se ressentent dès le niveau 1, mais avec beaucoup moins d'intensité. Des variations, moins amples encore, sont également enregistrées au niveau 3 et dans la Galerie des Enfants, située dans les anciens ateliers pédagogiques du 1er étage de la GGE. Ces espaces jouent-ils, même 
faiblement, un rôle "tampon" par rapport à l'extérieur, puisqu'ils sont en "périphérie" du bâtiment? Le gradient d'hygrométrie dans la nef s'établit ainsi en partie du bas vers le haut. Les courbes de température restent très stables, quel que soit le niveau considéré, même si les variations sont légèrement plus importantes aux niveaux 0 et 3 , pour les mêmes raisons que l'hygrométrie.

Les explosions populationnelles de mites semblent dues à des facteurs intérieurs et non soumis à variation aléatoire. Les relevés de climat extérieur (archives, www.meteociel.fr, site consulté en mai 2013), plutôt froids en 2013, ne permettent pas d'expliquer la situation.

\section{Hypothèse 3 : La poussière facteur favorable ou défavorable?}

Cette hypothèse est confortée par le constat d'un empoussièrement régulier et constant, déjà anticipé dès l'ouverture de la galerie en 1994 (Maigret et al., 2002). Depuis 20 ans, le bâtiment a accueilli plus de 11 millions de visiteurs.

Vingt-neuf prélèvements manuels de poussière ont donc été réalisés sur les 5 niveaux de la GGE (R0 à R+3 et Galerie des Enfants), tous hors vitrines, en des lieux soigneusement définis. Certains l'ont été à proximité des pièges à phéromones pour voir s'il y a une corrélation entre poussière importante et utilisation de cette ressource par les mites, d'autres à des endroits où la pression d'observation (directe ou par pièges) est moins importante. L'idée était de pouvoir obtenir un maillage entre observations directes (adultes morts ou vivants), pièges à phéromones, prélèvements de poussière.

Un exemple de répartition des pièges et des prélèvements : le niveau $R+2$ de la GGE. La flèche localise le prélèvement de poussière figuré ci-dessous.

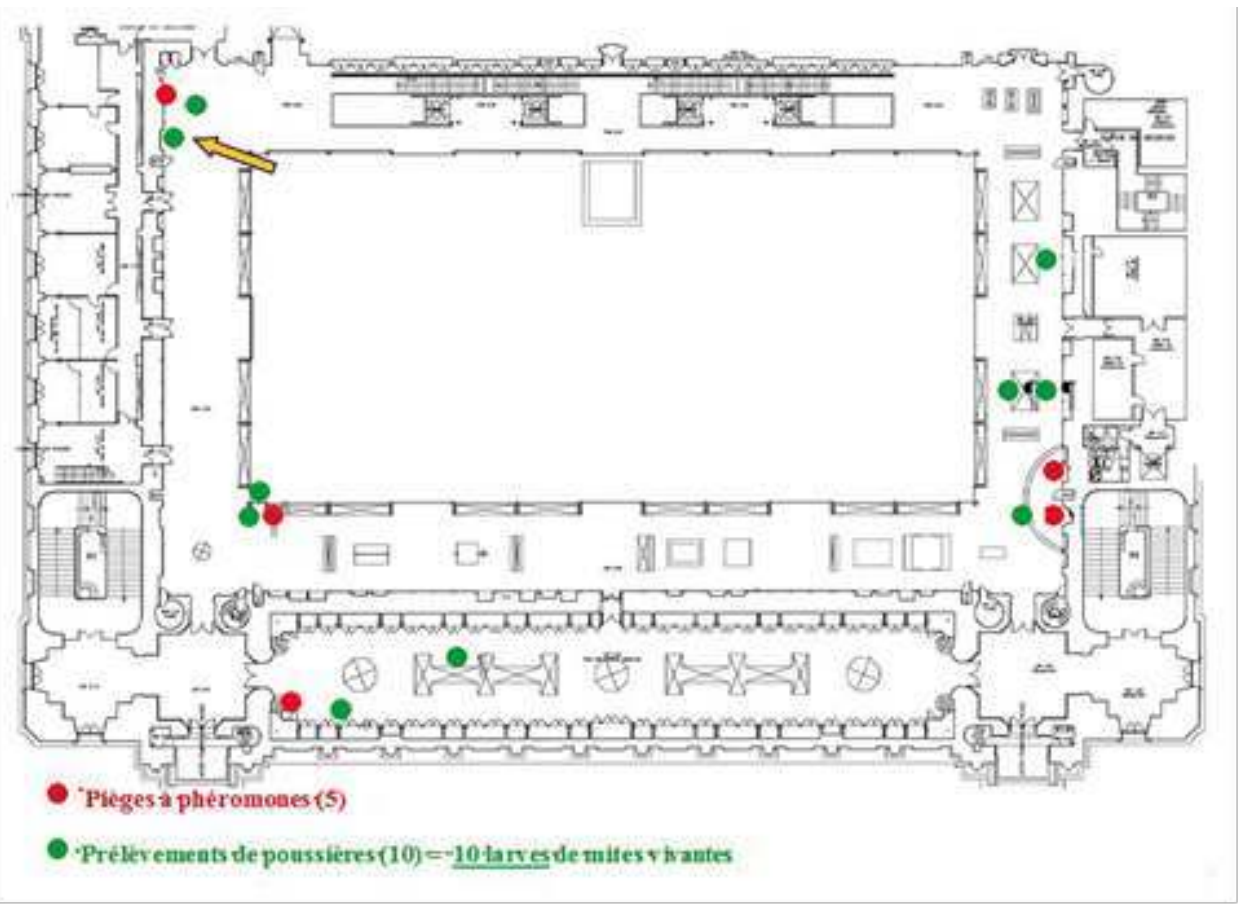

(c) MNHN 
prèlèvements ont tous été analysés sous loupe binoculaire, afin de rechercher compter et identifier les restes d'insectes qui s'y trouvaient (œufs, larves, nymphes, imagos, exuvies ou restes nymphaux). En plus de la poussière minérale, la totalité des prélèvements contenait de nombreux phanères animaux ou humains, beaucoup contenaient des restes d'alimentation, des fibres vestimentaires, autant de matériaux pouvant servir d'alimentation aux mites (Florian, 1997). À cinq exceptions près (arrière de bouches d'aération sous des vitrines et dessous du plancher de la caravane), les prélèvements $(n=24)$ contenaient des larves et des imagos vivants et/ou morts d'insectes appartenant à des ordres divers et, pour 14 d'entre eux, de Tineola bisselliella. Le nombre de larves décomptées s'élève à 61 , soit 2,1 larves/prélèvement. À ce nombre s'ajoutent 5 imagos, ce qui corrobore les captures abondantes des pièges.

Quatre larves vivantes sont décomptées. Des adultes ont été observés rentrant ou sortant de la base de ce podium, tout comme en d'autres secteurs, ou sous des vitrines et des balustrades. Ces zones interstitielles, difficilement accessibles sont de fait très poussiéreuses, et ne sont pas prises en compte dans les prestations d'entretien des espaces.

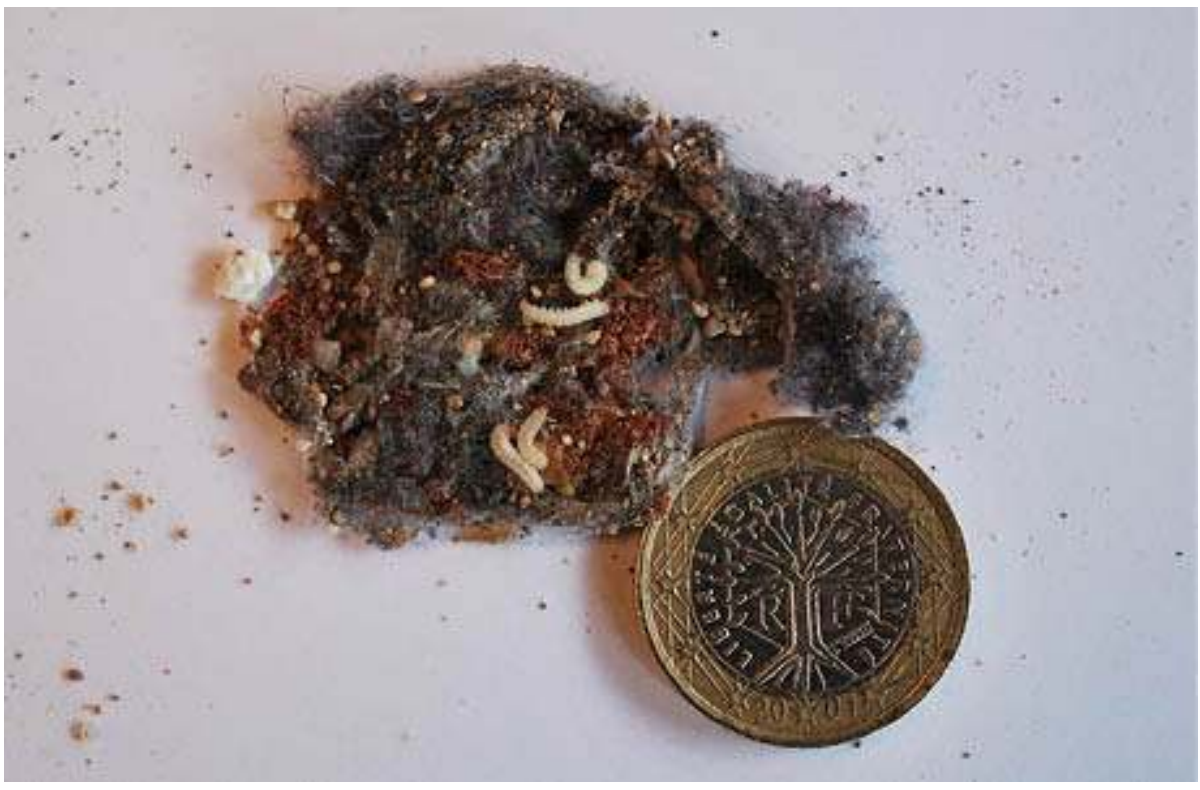

(c) MNHN

À la Grande Galerie de l'Évolution, le travail de maintenance et de dépoussiérage a été confié depuis plusieurs années à des prestataires. Les ressources allouées à ce poste de dépense diminuant, de nombreux lieux ne sont plus systématiquement nettoyés, voire sont totalement oubliés, comme les dessous des sièges. D'autres enfin, inaccessibles, échappent à peu près systématiquement à l'action de l'agent en charge du dépoussiérage ou du technicien cordiste. L'inspection de tous les endroits reculés et délaissés en termes de ménage permet de corroborer nos hypothèses de travail quant au rôle de l'empoussièrement dans la prolifération des insectes, la poussière agissant comme substrat nutritif, selon sa composition ou comme milieu de protection contre le froid. D'après les relevés et observations, cette poussière contient, selon les endroits, des débris de nourriture tels que miettes de biscuits, friandises... jusqu'à des morceaux de jambon et même une peau entière de banane restée pendant des mois sans être enlevée. Il faut insister sur le fait que, kératophages à tendance polyphage (Fraenkel et Blewett, 1946), les mites se nourrissent aussi bien de matériaux d'origine animale que végétale (chitine, 
poils, plumes, coton, soie, aliments contenant de l'amidon, débris végétaux...). Leur alimentation est continue durant le stade larvaire, sauf au moment de la mue, tandis que les adultes ne s'alimentent pas ou peu. Les larves sécrètent des enzymes particulières leur permettant de digérer plus spécifiquement la kératine (Florian, 1997 ; Robinson, 2004). Ces accumulations de composition variable contiennent en fait tous, en plus ou moins grande quantité, de la matière organique susceptible de servir de substrat nutritif aux larves de Tineola bisseliella. La plupart du temps ces larves se "contentent" de ces débris, mais réagissent immédiatement au placement de spécimens nouveaux et naturalisés sans mise en œuvre de composés ou dérivés d'arsenic. Tous les spécimens ainsi changés, même de manière temporaire, ont été ravagés de manière plus ou moins importante dans les semaines qui ont suivi leur installation, c'est-à-dire le temps pour les œufs d'éclore (oiseaux sur la caravane en remplacement d'un rhinocéros très fissuré, Vautour de l'Himalaya sur une corniche, oiseaux de "la Ferme de la domestication"...).

Plus surprenant, et en marge des prélèvements manuels, près de 90 mites adultes mortes ont également été décomptées dans les vitrines, théoriquement hermétiques, qui se trouvent aux niveaux 0 et 1 . Cette découverte, fortuite en juin 2013, fait l'objet d'une surveillance particulière et systématique depuis cette période, mais aucun nouvel intrus n'a été décompté depuis lors. Ces vitrines abritent les spécimens les plus sensibles et les plus fragiles (entomologie), dont aucun n'a toutefois été dégradé par une infestation. La question de la présence de ces mites a évidemment posé celle de l'étanchéité de ces vitrines. Par où ces individus sont-ils rentrés, alors que la granulométrie de la poussière à l'intérieur de ces vitrines montre des particules excessivement fines, de surcroît en quantité infime (ces vitrines ne sont jamais ouvertes)? Jusqu'alors, seules les vitrines anciennes, en bois et verre ou en métal et verre, dont les joints d'ouvrants n'avaient pas été changés/améliorés, étaient connues pour laisser passer poussière et insectes, et étaient surveillées en conséquence.

De fait, au niveau 0, les cailloux enchâssés dans du béton laissent des interstices importants, encore aujourd'hui tous remplis de poussière dans laquelle des larves de mites se développent en permanence (C. Gottini, communication personnelle, septembre 2014). Ces endroits, qui nécessiteraient un nettoyage de fond, encore jamais réalisé, doivent être considérés comme un "réservoir" populationnel, à l'abri de la lumière et du dérangement, et au pied de vitrines hermétiques. 
Une larve sur une friandise : celle de couleur verte, chocolatée, a été aussi attaquée. La pièce de un euro donne l'échelle : les larves de petite taille passent inaperçues dans la poussière.

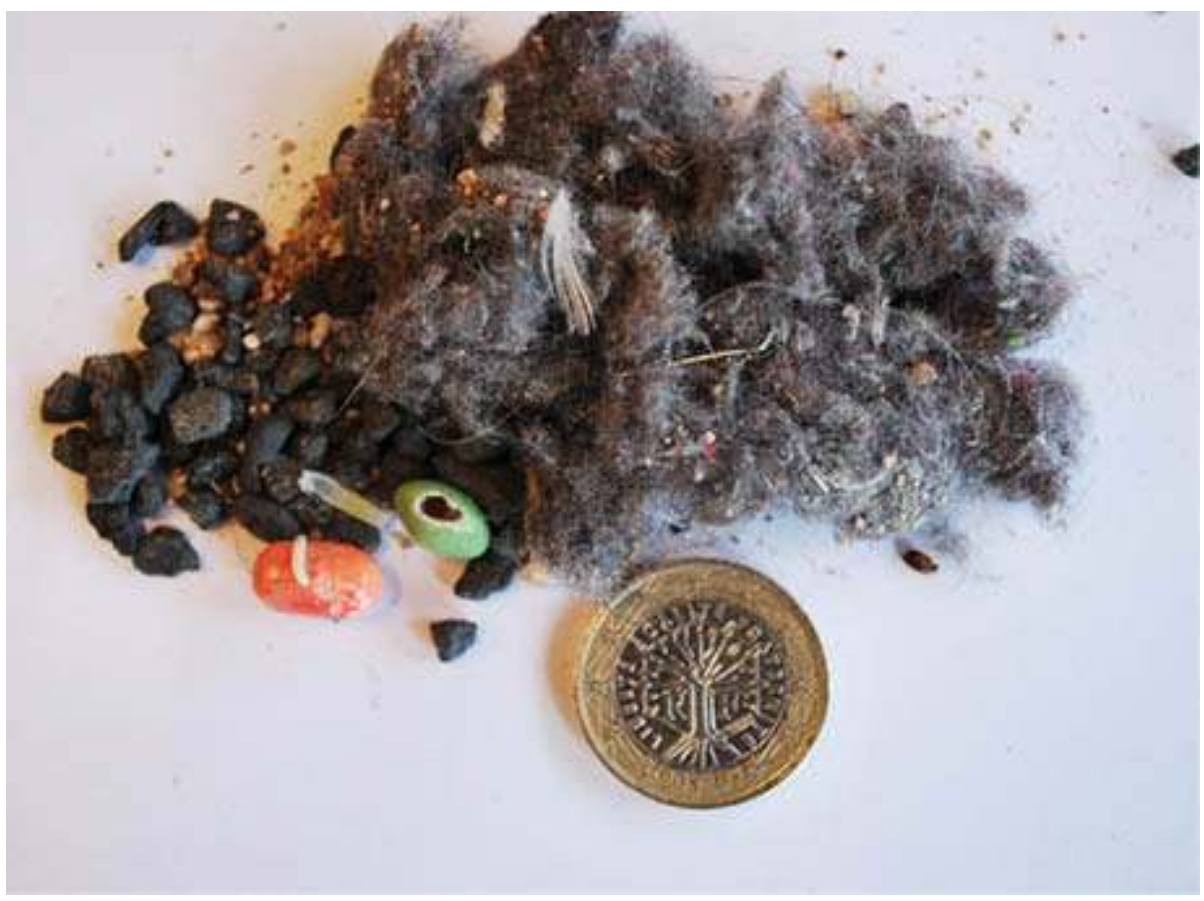

(c) $\mathrm{MNHN}$

Enfin, ces prélèvements de poussière ont également amené un résultat initialement non recherché, à savoir la découverte à tous les niveaux de la GGE, d'Attagène non spécifié (Attagenus sp., identification faite par l'unité de collections d'entomologie du MNHN en juin 2013), sous forme de restes (97 exuvies, 2 larves et 4 imagos morts, présence de restes dans 20 des 29 échantillons de poussière) ou d'individus vivants (14 larves). L'ampleur de cette présence constitue une nouveauté. La préoccupation principale des différents acteurs de la conservation des collections exposées s'étant portée sur les mites, il n'est pas illogique de penser que la détection systématique et organisée d'autres espèces de ravageurs a été moins accentuée. Le faible nombre de captures d'individus vivants lors de l'échantillonnage s'explique sans doute en partie par leur rapidité à fuir et au fait qu'ils restent souvent au ras du sol. L'absence d'utilisation de pièges ciblés renforce ce résultat.

\section{Discussion}

La poussière s'avère être donc l'hypothèse la plus probante quant à l'explication de la prolifération des mites à la GGE. Si cette conclusion était attendue, il est ici montré que cette poussière est un facteur majeur qui favorise la reproduction des insectes et le développement des populations à la GGE.

En effet, l'empoussièrement, s'il constitue pour le gestionnaire de collections un indicateur d'entretien inadapté ou incomplètement réalisé, peut également être regardé comme un indicateur de "quiétude", pour les insectes : la poussière laissée accumulée constitue en définitive un biotope particulièrement favorable aux Tineidae (poussière non enlevée = pas de dérangement, température préservée, capacité de dissimulation, réserve trophique). À un facteur écologique de grande disponibilité trophique s'ajoute 
donc un facteur écologique de domaine vital à faible pression de destruction (une désinsectisation annuelle seulement) et à faible voire nul facteur de perturbation dans certains secteurs (ceux non nettoyés,probablement, depuis des années).

Il est désormais certain que de telles quantités de ressources trophiques ont :

- permis le développement d'une population très importante de Tineidae ;

- que cette population à caractère insulaire à l'échelle de la Grande Galerie vit à peu près en autarcie au long de l'année, dans ce que l'on peut considérer comme un isolat géographique, pourtant non complètement fermé ;

- et peut-être le plus important en matière de conservation des spécimens exposés, que ces disponibilités trophiques abondantes ont "fixé" les mites sur la poussière plus que sur les spécimens eux-mêmes, réduisant ainsi les dégradations et les pertes. Le manque de ménage peut même (un comble) être considéré comme agissant de manière "bénéfique" sur les spécimens de fabrication récente, qui ne contiennent pas de dérivés ou composés arsenicaux et qui ne sont donc aucunement protégés de manière passive contre les attaques des kératophages. Autrement dit, la poussière contient suffisamment de ressources trophiques pour que les populations ne migrent pas entièrement vers d'autres sources de nourriture.

Le constat applicable à la GGE reste aujourd'hui celui d'un espace durablement infesté, à cause de conditions écologiques favorables aux Tineidae, conditions qui doivent être corrélées à la réduction progressive des moyens alloués à la maintenance du bâtiment y compris sa récente extension destinée au public junior, la Galerie des Enfants. Dans le même temps, la fréquentation du public a augmenté dans cet espace. Selon les chiffres disponibles (Ministère de la Culture, 2010, 2013), la croissance du public a été de plus de $35 \%$ entre 2009 et 2011, au moment précis du renouvellement du marché d'entretien des espaces, qui a vu la somme précédemment allouée à la seule Grande Galerie désormais attribuée à l'ensemble des locaux bâtis du MNHN... 
Les grues couronnées sur la caravane. L'infestation initiée sous le croupion, s'est traduite par la chute de débris de barbes et de barbules. L'infestation n'a été décelée que grâce à ce dégât et serait passée totalement inaperçue autrement.

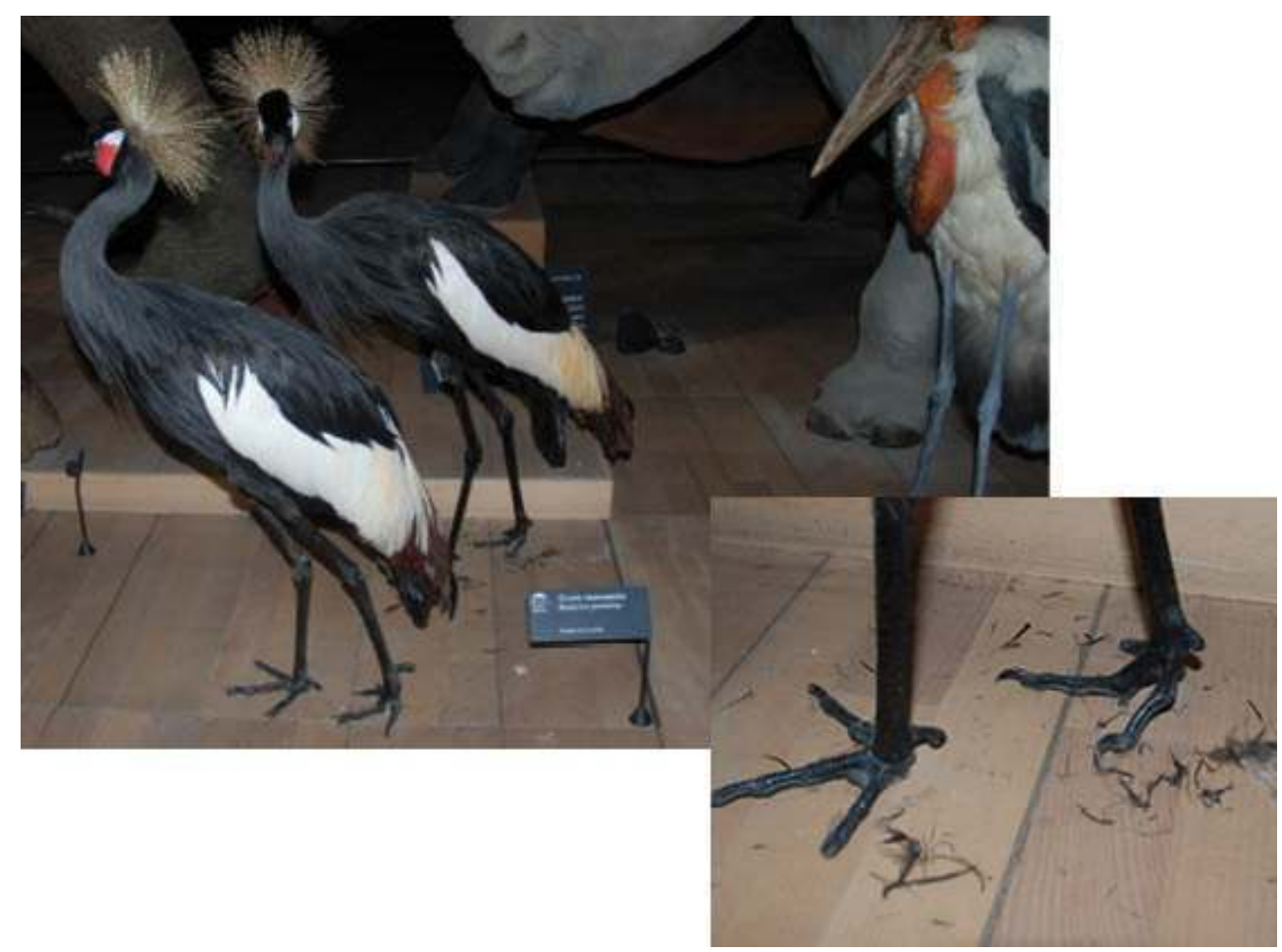

Mais toute nouvelle ressource est exploitée de manière opportuniste: l'exposition temporaire Nuit, ouverte au public le 12 février 2014 a connu une infestation progressive de mites dès les premiers jours d'exploitation : un relevé des quatre pièges à phéromones placés dans l'exposition, effectué le 24 février, indiquait déjà 12 captures... (Direction des Collections, communication personnelle). De même, des grues couronnées placées sur la caravane, après avoir été congelées à $-40^{\circ} \mathrm{C}$ (Pacaud, 1996), ont été attaquées en seulement quelques semaines. Voilà qui confirme, si besoin en est encore, que les attaques les plus importantes de ces dernières années concernent systématiquement des spécimens récents, nettement plus attirants : le cas de l'ouverture, en octobre 2010, de la Galerie des Enfants, ne déroge pas à cette règle, même si une surpression effective et efficace de ce petit volume, bien plus facilement maitrisable que la grande nef parce que petit, très bien fermé au moyen de portes bien étanches, a maintenu les mites à l'extérieur pendant deux ans... Seuls 2 individus ont été capturés pendant la durée de cette étude (printemps 2013), une augmentation du nombre de captures a depuis lors été remarquée. Les spécimens d'animaux présentés dans la Galerie des Enfants sont neufs pour la plupart (réalisés sans arsenic), et donc beaucoup plus facilement et plus rapidement "exploités" par les insectes kératophages.

La question de la surpression de la Grande Galerie de l'Évolution, régulièrement invoquée dès que l'on parle de lutte contre les insectes dans cet espace, semble toutefois n'avoir jamais réellement été efficace (Gagnier et al., 1997), ce que l'on comprend aisément au vu de l'immensité de la nef, augmenté du nombre d'ouvertures, salles annexes, couloirs, gaines techniques débouchant sur l'extérieur... qui en amoindrissent la performance. Bien maitrisée, cette technique pourrait avoir un effet réel répulsif, mais l'expérience de ces 20 
dernières années ne permet malheureusement pas d'être affirmatif à cet égard. Son inefficacité compte au nombre des facteurs ayant favorisé l'apparition et le maintien des populations de mites à la Grande Galerie.

\section{Conclusion}

Cette étude a renforcé l'idée première que le piégeage, à but à la fois préventif et informatif, permet de surveiller l'évolution du nombre de Tineola bisselliella et d'intervenir rapidement si une explosion du nombre de captures a lieu. Le piégeage est donc une véritable composante du pilotage IPM (Integrated Pest Management), y compris dans des espaces complexes ${ }^{1}$.

La question qui se pose désormais à la GGE est celle de la réduction de la population de Tineidae, son éradication totale semblant une gageure au vu de l'efficacité très modérée des traitements chimiques possibles en ERP de nos jours. À cela s'ajoute la performance réduite des traitements, dans la mesure où la poussière constitue un obstacle à la dispersion des produits de traitement en même temps qu'un lieu de développement privilégié des larves. Ces deux facteurs combinés peuvent avoir contribué à ce que les insectes aient développé des résistances aux pyréthrinoïdes mis en œuvre. Une étude sur ces éventuels phénomènes de résistance est évoquée depuis plusieurs années maintenant, puisque le problème se pose pour toutes les collections à composante kératine/chitine du Muséum (ainsi que dans d'autres collections comparables), mais n'a pour l'instant recueilli aucun écho de la part des partenaires potentiels contactés. Or, il nous semble absolument nécessaire de pouvoir mener une expérimentation destinée à mesurer la résistance des populations de mites aux moyens chimiques actuellement autorisés dans les musées. Une telle étude servirait en effet à d'autres établissements ouvrant des espaces de présentation permanente de collections à dominante organique animale (naturalisation, textiles...), qui seraient susceptibles de connaître les mêmes inconvénients. Elle orienterait le choix des traitements et des stratégies.

Comme dans d'autres domaines, le salut peut peut-être venir du croisement de plusieurs approches: leurres chimiques, traitement direct, entretien et dépoussiérage accru. La lutte biologique est à l'étude, même si certains retours d'expérience semblent mitigés (A. Préviato, communication personnelle; Anheuser et Garcia Gomez, 2013). Dans tous les cas, l'entretien régulier du bâtiment en termes de ménage précis est une évidence et même la première condition : il ne sert à rien de dépenser des sommes importantes en traitements divers sans ménage préalable.

39 Cela étant la performance des traitements et protocoles appliqués devrait pouvoir être mesurée par ce qui pourrait être exprimé sous la forme d'un "indice de salubrité", indicateur d'un plan de prévention large contre les destructeurs kératophages, applicable à d'autres espaces de collections (armoire, salle voire bâtiment de réserve en son entier... ). La mise au point d'un tel indice relève d'une étude spécifique ultérieure.

L'éradication des insectes kératophages, si elle était menée à la GGE, serait de toute manière non pérenne, au vu de la porosité du bâtiment (indépendamment du public), de la diversité des activités qui s'y déroulent (la GGE est aussi devenue en 20 ans une forme d'open-space pour événementiels à large spectre culturel) et des procédures diverses qui en régissent le fonctionnement. La récente mise en place (avril-mai 2014) d'un nouveau protocole de ménage appliqué aux spécimens exposés est annoncée comme un pas 
important dans cette lutte de longue haleine. Ce nouveau protocole voit la mise en place de deux agents relevant directement et exclusivement de la Direction des Collections, agents qui ne s'occupent, en fonction du temps qui leur est alloué, que du ménage des collections exposées, à l'exclusion de toute autre tâche (surveillance des pièges à phéromone par exemple). Leur action est cependant quotidienne dans la limite de 5 jours par semaine, et consignée de manière à ce que leur responsable puisse effectuer un suivi et évoquer une stratégie de lutte avec les acteurs concernés par ce dossier. La consignation quotidienne permet de plus une réaction très immédiate, ce qui est un facteur de succès dans la lutte contre les ravageurs de collection.

\section{BIBLIOGRAPHIE}

Anheuser, K. et Garcia Gomez, I. Trichogramma evanescens contre Tineola bisseliella : expérience de lutte biologique contre la mite des vêtements dans une réserve d'objets ethnographiques. Studies in Conservation, vol. 58, $\mathrm{n}^{\circ} 3$, pp. 269-273.

Chauvin, G. Contribution à l'étude des insectes kératophages (Lepidoptera - Tineidae) : leurs principales adaptations à la vie en milieu sec. Thèse, université de Rennes, 1977, 294 p.

Chauvin, G. et Vannier, G. Supercooling capacity of Tineola bisselliella (Hummel) (Lepidoptera : Tineidae) : Its implication for disinfestation, Elsevier Science, n 4, 1997, pp. 283-287.

Florian, M-L. E. Heritage eaters. London : James \& James, 1997, 164 p.

Fohrer, F. Le diagnostic des infestations en milieu patrimonial : approches techniques et méthodologiques, La Lettre de l'OCIM, $\mathrm{n}^{\circ}$ 138, 2011, pp. 31-40.

Fraenkel, G. et Blewett, M. The dietetics of the clothes moth Tineola bisselliella Hum, Journal of experimental Biology, 1946, pp. 156-161.

Gagnier, P.-Y., Dennys, V. et Maigret, J. Le processus de mise en place de la régulation des températures et de l"hygrométrie dans un bâtiment rénové ou : un problème peut en cacher d'autres. In La conservation : une science en évolution. Actes des 3es Journées internationales d'études de l'Arsag, 1997, pp. 62-69.

Maigret, J., Gagnier, P.-Y. et Jullien, F. La lutte contre les insectes dans la Grande Galerie de l'évolution, in Les contaminants biologiques des biens culturels. Paris : Muséum national d'Histoire naturelle et Elsevier, "Collection Patrimoine", 2002, pp. 215-221.

Ministère de la Culture. Muséostat 2006. 2007, 44 p. (disponible sur www.culture.gouv.fr/culture/ politique culturelle/).

Ministère de la Culture. Chiffres clés - Statistiques de la Culture. Éditions de 2010 et de 2013, 14 p. (disponibles sur www.culturecommunication.gouv.fr/etudes-et-statistiques).

Pacaud, G. La désinsectisation par le froid, La Lettre de l'OCIM, n 47, 1996, pp. 31-32.

Robinson, G.-S. Moth and Bird interactions : guano, feathers and detritophagous caterpillars (Lepidoptera : Tineidae), in Van Emden, H. and Rothschild, M. Insect and Bird interactions. Andover, Hampshire : Intercept, 2004. 
Strang, T.-J. K. A review of published temperatures for the control of pest insects in museums, Collection Forum, 1992, 8(2), pp. 41-67.

\section{NOTES}

1. L'IPM est une stratégie de lutte contre les insectes ravageurs fondée sur l'écosystème qui met l'accent sur la prévention à long terme contre les ravageurs ou les dégâts grâce à une combinaison de techniques comme la lutte biologique, la manipulation de l'habitat, la modification des pratiques culturelles, et l'utilisation de variétés résistantes.

\section{RÉSUMÉS}

La préparation des 20 ans de la Grande Galerie de l'Évolution a été l'occasion d'établir un diagnostic synthétique des infestations passées et actuelles qui frappent les collections en exposition permanente : conçue comme une aide à la définition de la stratégie de lutte, cette démarche passe en revue les principales hypothèses retenues pour expliquer ces phénomènes.

\section{INDEX}

Mots-clés : infestation, exposition permanente, Grande Galerie de l'Evolution

\section{AUTEURS}

\section{BENOÎT GAYRAL}

Etudiant en Master 2 Muséologie : Sciences, Cultures et Sociétés au Muséum national d'Histoire naturelle

gayral.ben@gmail.com

\section{JACQUES CUISIN}

Responsable de la plateforme de préparation et restauration, direction des Collections, Muséum national d'Histoire naturelle cuisin@mnhn.fr 